\title{
GALOIS ENDOMORPHISMS OF THE TORSION SUBGROUP OF ONE-PARAMETER GENERIC FORMAL GROUPS
}

\author{
KARL ZIMMERMANN
}

(Communicated by William C. Waterhouse)

\begin{abstract}
Let $\mathbf{Z}_{p}$ be the ring of $p$-adic integers and let $\Gamma$ be a one-parameter generic formal group of finite height $h$ defined over $\mathbf{Z}_{p}\left[\left[t_{1}, \ldots, t_{h-1}\right]\right]=A$. Let $K$ be the field of fractions of $A, G=\operatorname{Gal}(\bar{K} / K)$ and $T(\Gamma)$ the Tate module of $\Gamma$. The purpose of this paper is to give an elementary proof that the map $\operatorname{End}_{A}(\Gamma) \rightarrow \operatorname{End}_{G}(T(\Gamma))$ is a surjection.
\end{abstract}

1. Introduction. In [4], using some deep results from class field theory and algebraic geometry, Tate proved the following

THEOREM. Let $R$ be an integrally closed, noetherian, integral domain whose field of fractions $K$ is of characteristic zero. Let $F$ and $H$ be p-divisible groups defined over $R$, and let $T(F), T(H)$ be the Tate modules of $F$ and $H$ respectively. The map $\operatorname{Hom}_{R}(F, H) \rightarrow \operatorname{Hom}_{G}(T(F), T(H))$ is bijective, where $G=\operatorname{Gal}(\bar{K} / K)$.

Subsequently, in [2], Lubin presented a short, elementary proof of a special case of the above theorem which applies to certain one-parameter formal groups defined over the ring of integers in a finite dimensional extension of the field of $p$-adic numbers. In this paper, we will show that Lubin's method of proof can be used in a slightly more general situation which will apply directly to one-parameter generic formal groups of height $h, \Gamma_{t_{1}, \ldots, t_{h-1}}(x, y)$ defined over $\mathbf{Z}_{p}\left[\left[t_{1}, \ldots, t_{h-1}\right]\right][[x, y]]$ where $\mathbf{Z}_{p}$ is the ring of $p$-adic integers. (See [1] for definitions and notation regarding generic formal groups.) In particular, we will prove

THEOREM 1. Let $A$ be a complete regular local ring of characteristic 0 with residue field $k$ of characteristic $p>0$. Let $K=\operatorname{Frac}(A)$ be the field of fractions of $A$ and $\bar{K}$ an algebraic closure of $K$ with $G=\operatorname{Gal}(\bar{K} / K)$. Let $F$ be a one-parameter formal group defined over $A$, of finite height $h$, that has $f \in \operatorname{End}_{A}(F)$ such that $f^{\prime}(0)$ is a parameter of $A$. If $\phi$ is a $G$-endomorphism of the group $\Lambda(F)$ of points of finite order of $F$ then there exist $g \in \operatorname{End}_{A}(F)$ such that for every $\lambda \in \Lambda(F)$, $g(\lambda)=\phi(\lambda)$.

Parts of the proof are based on properties of regular local rings (see for example [3]). Specifically we will refer to

(*) If $(A, M)$ is a regular, local ring and $f(x) \in A[x]$ satisfies $f(x)=x^{n}+$ $a_{n-1} x^{n-1}+\cdots+a_{1} x+a_{0}$ with $a_{i} \in M$ and $a_{0} \in M \backslash M^{2}$, then $f(x)$ (which is called an Eisenstein polynomial) is irreducible over the field of fractions of $A$. Moreover, if

Received by the editors October 12, 1986.

1980 Mathematics Subject Classification (1985 Revision). Primary 14L05; Secondary 13 H05.

Key words and phrases. One parameter formal group, local ring.

This paper appears as part of the author's dissertation written at Brown University. 
$f(\alpha)=0$ then $A[\alpha]$ is regular local ring and if $N$ is the maximal ideal, $\alpha \in N \backslash N^{2}$, i.e. $\alpha$ is a parameter of $A[\alpha]$.

Continuing with preliminary notions, if we now take $A$ to be complete in the $M$-adic topology, the Weierstrass preparation theorem applies. More precisely, if $f(x) \in A[[x]]$ with wideg $(f)<\infty^{1}$ and $f(0)$ a parameter of $A$, then $f(x)=P(x) u(x)$ where $P(x)$ is an Eisenstein polynomial in $A[x]$ and $u(x)$ is a unit in $A[[x]]$. Such an $f(x)$ will be called an Eisenstein power series. Clearly $f(x)$ is indecomposable in $A[[x]]$, and in $\bar{K}$ (an algebraic closure of $K=\operatorname{Frac}(A)), f(x)$ has wideg $(f)$ distinct roots which form a complete set of conjugates over $K$.

Suppose $F$ is a one-parameter formal group defined over the complete, regular, local ring $A$ (with field of fractions $K$ ). Let $\Lambda(F)=\bigcup_{m=1}^{\infty}$ (zeros in $\bar{K}$ of $\left[p^{m}\right]_{F}(x)$ ), and note that the set $\Lambda(F)$ can be endowed with a group structure with addition defined as follows: if $\alpha, \beta \in \Lambda(F), \alpha+_{F} \beta=F(\alpha, \beta)$. This substitution makes sense because $\alpha$ and $\beta$ are nonunits in $A[\alpha, \beta]$ which is finite as an $A$-module, hence complete. The formal group endomorphism $\left[p^{m}\right]_{F}$ induces a group endomorphism $\left[p^{m}\right]_{F}: \Lambda(F) \rightarrow \Lambda(F)$ defined by $\lambda \rightarrow\left[p^{m}\right]_{F}(\lambda)$. It is clear that $\operatorname{ker}\left(\left[p^{m}\right]_{F}\right)$ is equal to the set of zeros of $\left[p^{m}\right]_{F}(x)$ in $\bar{K}$.

2. Proof of Theorem 1. The notation to be used was introduced in the statement of Theorem 1. The first step in the proof is to find a power series $g \in A[[x]]$ such that for every $\lambda \in \Lambda(F), g(\lambda)=\phi(\lambda)$. The reader is referred to remarks in Lubin [2] to see that it is enough to find a power series $g_{n} \in A[[x]]$ for each $n$ such that for every $\lambda \in \operatorname{Ker}\left[p^{n}\right]_{F}, \phi(\lambda)=g_{n}(\lambda)$. To that end, let $\alpha$ be a root of the Eisenstein power series $f(x) / x, K(\alpha)=L$ and $B$ the ring of integers in $L$. Recalling $(*)$, it is clear that $A[\alpha]$ is a regular local ring (hence integrally closed) and $\alpha$ is a parameter. Moreover, since $\operatorname{Frac}(A[\alpha])=K(\alpha)=L$, it may be concluded that $A[\alpha]=B$. We note that $\left[p^{n}\right]_{F}(x)-\alpha \in B[[x]]$ is an Eisenstein power series and if $\beta$ is a root of this power series then a complete set of $L$-conjugates of $\beta$ is given by $\beta+{ }_{F} \operatorname{ker}\left[p^{n}\right]_{F}$.

Now consider the tower of fields $K \subseteq L \subseteq L(\beta) \subseteq \bar{K}$. Let $H=\operatorname{Gal}(\bar{K} / L(\beta))$, and note that for $\sigma \in H, \sigma(\phi(\beta))=\phi(\sigma(\beta))=\phi(\beta)$. We see that $\phi(\beta)$ is in the fixed field of $H$, namely $L(\beta) . \phi(\beta) \in \Lambda(F)$ by definition and so $\phi(\beta) \in L(\beta) \cap \Lambda(F)$. However, using $(*)$, we see that the ring of integers in $L(\beta)$ is $B[\beta]$. Consequently, $\phi(\beta) \in L(\beta) \cap \Lambda(F) \subseteq B[\beta]$ implies there exists $\gamma(x) \in B[x]$ with $\gamma(\beta)=\phi(\beta)$. Clearly $\gamma(0)$ is a nonunit.

At this point we note that the remarks thus far will allow us to apply Lubin's methods directly to our more general situation. Specifically, define $g_{*}(x) \in B[\beta][[x]]$ by $g_{*}(x)=\gamma\left(\beta+{ }_{F} x\right)-{ }_{F} \phi(\beta)$ and observe that for $\lambda \in \operatorname{ker}\left[p^{n}\right]_{F}$,

$$
g_{*}(\lambda)=\gamma\left(\beta+{ }_{F} \lambda\right)-{ }_{F} \phi(\beta)=\phi\left(\beta+{ }_{F} \lambda\right)-{ }_{F} \phi(\beta)=\phi(\lambda) .
$$

The second equality follows because $\beta+_{F} \lambda$ is an $L$-conjugate of $\beta$; thus there exists $\sigma \in \operatorname{Gal}(\bar{K} / L)$ with $\sigma(\beta)=\beta+{ }_{F} \lambda$. Therefore

$$
\phi\left(\beta+{ }_{F} \lambda\right)=\phi(\sigma(\beta))=\sigma(\phi(\beta))=\sigma(\gamma(\beta))=\gamma(\sigma(\beta))=\gamma\left(\beta+_{F} \lambda\right) .
$$

By the Weierstrass preparation theorem,

$$
\left[p^{n}\right]_{F}(x)=x P_{n}(x) u_{n}(x) \quad \text { where } \operatorname{deg} P_{n}(x)=p^{n h}-1
$$

${ }^{1}$ Let $\bar{f}(x)$ be obtained by reducing the coefficients of $f \operatorname{modulo} M$. Wideg $(f)=n$ if and only if $\bar{f}(x)=\bar{a}_{n} x^{n}+\cdots+\bar{a}_{0}, \bar{a}_{n} \neq 0$. 
This induces an isomorphism between the rings $B[\beta][[x]] /\left(\left[p^{n}\right]_{F}(x)\right)$ and $B[\beta][x] /\left(x P_{n}(x)\right)$ and the isomorphism yields $g_{n}(x) \in B[\beta][x]$ with $g_{*}(x) \cong g_{n}(x)$ $\bmod \left[p^{n}\right]_{F}(x)$ and $\operatorname{deg}\left(g_{n}(x)\right)<p^{n h}$. If $\sigma \in G$ then for every $\lambda \in \operatorname{ker}\left[p^{n}\right]_{F}$

$$
\left(g_{n}(\lambda)\right)=\sigma\left(g_{*}(\lambda)\right)=\sigma(\phi(\lambda))=\phi(\sigma(\lambda))=g_{*}(\sigma(\lambda))=g_{n}(\sigma(\lambda)) .
$$

However, $\operatorname{deg}\left(g_{n}^{\sigma}(x)-g_{n}(x)\right)<p^{n h}$; but for every $\sigma \in G, g_{n}^{\sigma}(x)-g_{n}(x)$ has $p^{n h}$ roots and so $g_{n}^{\sigma}(x)=g_{n}(x)$. Thus $g_{n}(x) \in A[[x]]$ as desired. Letting $g(x)=\lim g_{n}(x)$ yields $g(x) \in A[[x]]$ and for every $\lambda \in \Lambda(F), g(\lambda)=\phi(\lambda)$.

Note now that the power series $g \circ[p]_{F}(x)-[p]_{F} \circ g(x)$ has an infinite zero set (since $\Lambda(F)$ is infinite), whence $g \circ[p]_{F}=[p]_{F} \circ g$ which implies $g \in \operatorname{End}_{A}(F)$. Q.E.D.

COROLLARY 1. Let $\Gamma_{t_{1}, \ldots, t_{h-1}}(x, y) \in \mathbf{Z}_{p}\left[\left[t_{1}, \ldots, t_{h-1}\right]\right][[x, y]]=A$ be a generic formal group of height $h$. Let $\bar{K}$ be an algebraic closure of $\operatorname{Frac}(A)=K$ and $\phi$ a $\operatorname{Gal}(\bar{K} / K)$ endomorphism of $\Lambda\left(\Gamma_{t_{1}, \ldots, t_{h-1}}\right)$. Then there exists $g \in \operatorname{End}_{A}\left(\Gamma_{t_{1}, \ldots, t_{h-1}}\right)$ such that for all $\lambda \in \Lambda\left(\Gamma_{t_{1}, \ldots, t_{h-1}}\right), g(\lambda)=\phi(\lambda)$.

PROOF. $A$ is a complete regular local ring of characteristic 0 with residue field characteristic $p>0$. The endomorphism $[p]_{\Gamma_{t_{1}, \ldots, t_{h-1}}}(x)=f(x)$ has the property that $f^{\prime}(0)=p$, a parameter of $A$. Q.E.D.

We remark that the claim made in the abstract of this paper is an easy consequence of Corollary 1.

Finally, we observe that as is the case in Lubin [2], the proof of Theorem 1 yields the following result which is stronger (when it applies) than the result in [4].

COROLlaRY 2. If $A$ and $G$ are as in the statement of the theorem, and if $\phi$ is a $G$-endomorphism of $\operatorname{ker}\left[p^{n+1}\right]_{F}$, then the restriction of $\phi$ to $\operatorname{ker}\left[p^{n}\right]_{F}$ is analytic, i.e. there exists $f(x) \in A[[x]]$ such that for all $\lambda \in \operatorname{ker}\left[p^{n}\right]_{F}, \phi(\lambda)=f(\lambda)$.

The author would like to thank Professor Jonathan Lubin for many enlightening discussions on the subject of formal groups and for his helpful suggestions concerning this paper.

\section{BIBLIOGRAPHY}

1. J. Lubin and J. Tate, Formal moduli for one parameter formal Lie groups, Bull. Soc. Math. France 94 (1966), 49-60.

2. J. Lubin, Galois endomorphisms of the torsion subgroup of certain formal groups, Proc. Amer. Math. Soc. 20 (1969), 229-231.

3. M. Nagata, Local rings, Interscience, New York, 1962.

4. J. Tate, p-divisible groups, Proc. Dreibergen Summer School on Local Fields, 1966, SpringerVerlag, Berlin and New York, 1967.

Department of Mathematics, Union College, Schenectady, New York 12308 